Article

\title{
Joint Configuration and Scheduling Optimization of a Dual-Trolley Quay Crane and Automatic Guided Vehicles with Consideration of Vessel Stability
}

\author{
Lijun Yue, Houming Fan * and Chunxin Zhai \\ Department of Transportation engineering, Dalian Maritime University, Dalian 116026, China; \\ Yuelj11@163.com (L.Y.); cxzhai@dlmu.edu.cn (C.Z.) \\ * Correspondence: fhm468@dlmu.edu.cn; Tel.: +86-0411-8472-5868
}

Received: 28 November 2019; Accepted: 15 December 2019; Published: 18 December 2019

\begin{abstract}
This study proposes a formulation to optimize operational efficiency of a dual-trolley quay crane and automatic guided vehicles (AGVs) to reduce energy consumption at an automated container terminal. A two-phase model is used to minimize energy consumption during loading and discharging operations, as well as maximize the utilization rate of the AGVs, with consideration of relevant constraints such as the capacity of buffers for the quay crane (QC) and yard, the stability of vessel, the maximum endurance of an $\mathrm{AGV}$, and the available laytime for handling. We propose a constrained partial enumeration strategy to construct quay crane schedules and a genetic algorithm to solve the AGV scheduling problem. Finally, Yangshan Phase IV automated container terminal's data is used to verify the validity and applicability of the proposed model. The results of the tests provide evidence that the proposed method can improve energy efficiency.
\end{abstract}

Keywords: dual-trolley quay crane; AGV scheduling; energy consumption; available laytime; vessel stability

\section{Introduction}

To date, the London Port, Kawasaki Port, Singapore Port, Hamburg Port, Xiamen Ocean Gate Container Terminal, Shanghai Yangshan Port, and other ports have successively built automated container terminals. To remain competitive, in terms of services and prices, some companies and researchers have focused on optimizing the line's schedule reliability and the scheduling of handling equipment in container terminals. Sea Intelligence in Denmark reported in a study, in June 2019, that the Hyundai merchant ship ranked first on the list, with an average rate of $91.8 \%$. In the shipping alliance, the $2 \mathrm{M}^{\prime} \mathrm{s}$ schedule reliability is $91.3 \%$, which is the highest, while the Alliance is the lowest rate, at only $75.4 \%$. In terms of container terminals, increasingly, the emphasis has been on improving reliability by completing handling operations within laytime. In addition, efforts have been made to optimize the loading and discharging operations, which accounts for $48.3 \%$ of the energy consumption in a terminal [1]. The sequence of quay crane moves affects the stability and the scheduling of quay cranes influences the handling time of the ship. The arrival time of the AGV affects the loading and discharging containers by the quay crane and the yard crane (YC) [2]. Thus, this paper optimizes the configuration and scheduling of quay cranes and AGVs aiming to reduce energy consumption at an automated container terminal, with consideration of the reliability and vessel stability.

There are extensive literatures on the reduction of energy consumption at a seaport. A major category of container terminal operations includes the quayside operations, which contains two separate tasks, allocation of berths and quay crane scheduling. For example, Chang [3] proposed and verified an investigation about the scheduling strategy of berth and quay crane through a case 
analysis. He [4] developed a mixed integer model of berth allocation and quay crane allocation with the goal of minimizing the delay of ships and energy consumption of operations, and solved the model using the integrated simulation algorithm. Some researchers studied the scheduling of QC under a known berth plan, with the goal of minimizing makespan among all ships. For example, Chang [5] studied quay crane scheduling under dynamic ship arrival conditions, and proposed a dynamic rolling strategy, establishing a model to minimize the handling time of all ships in the port and balance the quay crane operation. Kovalyov [6] proposed five strategies to optimize the multiple quay crane scheduling problem of simultaneous loading and discharging operations. Other researchers have evaluated the effect of quay crane scheduling on reducing energy consumption by viewing a single container as a group. Liu [7] studied the allocation and scheduling of a quay crane and established an AGV queuing model with the goal of minimizing $\mathrm{CO}_{2}$ emissions during discharging. By analyzing the earliest workable time and required completion time of each task, a coupling model of quay crane scheduling and configuration was established by Liang [8], and used the loop iterative method to solve the model. Kovalyov [9] proposed an algorithm that included a cutting-plane and graph-based binary search to solve the exact solution of the quay crane scheduling model, and verified the effectiveness of the model and algorithm under different sized tasks. Kim [10] established a mixed integer programming model that considered the priorities of tasks and the safety margin between quay cranes, and proposed a greedy random adaptive algorithm based on branch and bound to solve the model. Nguyen [11] used a genetic algorithm and a genetic programming algorithm to solve the priorities of containers, respectively, to obtain a relatively stable makespan under an uncertain environment. Zhang [12] established a scheduling model with stability constraints that considered the ship's longitudinal stability during loading and discharging, and proposed a heuristic algorithm based on a sliding window to repair the sequence violating the stability constraints. Al-Dhaheri [13] developed a model to solve large-scale problems effectively and a Lagrangian relaxation to decompose problems that were not constrained by stability.

Another major equipment of container terminal operations is the AGV, which receives the discharged container from the quay crane and sends it to the yard or delivers the loaded container from the yard to the quay crane. Regarding the problem of reducing energy consumption of AGV operations, some researchers have studied the scheduling strategy. For example, Kim [14] aimed to reduce the delay energy consumption of ships at automated container terminals. Choe [15] proposed the ONPL (online preference learning) algorithm, which dynamically adjusted the scheduling strategy of an AGV by updating the preference function. Kim [16] studied the AGV scheduling problem by considering the multi-standard scheduling strategy. Some researchers have addressed the impact of the uncertain environment of AGV transportation. For example, Singgih [17] studied the AGV path planning problem of automated container terminals with consideration of congestion. An integer programming model was constructed with the objective of minimizing the transiting time and waiting time of the AGV and solved it using the Dijkstra algorithm. Legato [18] built an optimization model to minimize the collision time caused by the AGV path conflict, with consideration of the non-constant efficiency of the quay crane, and solved it using a simulated annealing algorithm.

More recently, several researchers have adopted integration as a means of simultaneously making decisions for multiple quay crane and AGV scheduling problems. Xin [19] proposed a two-part hierarchical control structure, which considered the operation to be continuous dynamics, as well as discrete dynamics, minimizing the completion time of the upper quay crane and the energy consumption of the lower layer AGV. Peng [20] quantified the impact of equipment configuration on carbon emissions, and established a simulation model based on complex queuing networks to optimize the ratio of the quay crane, the yard crane, and AGVs. On the basis of the hybrid flow shop scheduling problem (HFSS), Yang [21] proposed the dual-objective joint optimization model for a quay crane, truck, and yard crane with the goal of minimizing total operation time and energy consumption. Al-Dhaheri [22] proposed an optimization model aiming at minimizing the makespan of quay crane and the number of AGVs and solved the model with Cplex. Yang [23] studied the 
integrated scheduling of quay crane, $\mathrm{AGV}$, and yard crane for simultaneous loading and discharging operations, and designed a general algorithm based on preventive congestion rules. Xin [24] proposed a collision-free scheduling algorithm to generate timetables for AGV, quay crane, and yard crane, which could reduce the average distance of AGV operation.

In summary, the above literatures have incorporated either no equipment scheduling decisions or very simple equipment scheduling decisions in their models. In this study, we propose a model to optimize the joint configuration and scheduling of a dual-trolley quay crane and AGVs. The contributions of this study are threefold. First, the effects of available laytime are proposed and evaluated from the terminal system perspective. Second, the constraints of the buffer platform capacity of the dual-trolley quay crane and blocks are considered during configuration and scheduling. In this manner, this study fills the gap in the existing literature, which has evaluated the scheduling of AGVs only based on known time windows. Third, the utilization of an AGV is improved over the state-of-the-art in terms of the objective of energy consumption. In addition, this work is the first study to optimize the joint configuration and scheduling of multiple handling operations.

The remainder of this paper is organized as follows: Section 2 presents a two-phase model with the constraint of vessel stability, available laytime, AGV's endurance time, and buffer platform for quay crane and blocks. Section 3 describes the enumeration algorithm and genetic algorithm to solve the problems for seeking a satisfactory solution of AGV and quay crane scheduling. Section 4 proposes a real case to verify the validity of the model and algorithms and Section 5 summarizes the research results and proposes future research directions.

\section{Problem Description and Mathematical Model}

\subsection{Problem Description}

The loading and discharging process of an automated container terminal is shown in Figure 1. During the discharging period, the main trolley of the dual-trolley quay crane places the discharged container onto the transfer platform, and the gantry trolley takes the container from the transfer platform and places it on the AGV and, then, the AGV transports the container to the yard and places the container on the buffer bracket. During the loading period, the no-load AGV takes the loaded container from the buffer bracket of the yard to the underside of the gantry trolley and, then, the gantry trolley lifts the container from the AGV onto the transfer platform. When the main trolley idles, the container on the transfer platform is placed on the ship. In order to minimize the energy consumption of the loading and discharging operations, the following constraints must be considered by the dispatching center: available laytime of the ship in port, the stability of the ship, the precedence of tasks, a safety margin between quay cranes, the buffer size, and the AGV's endurance.

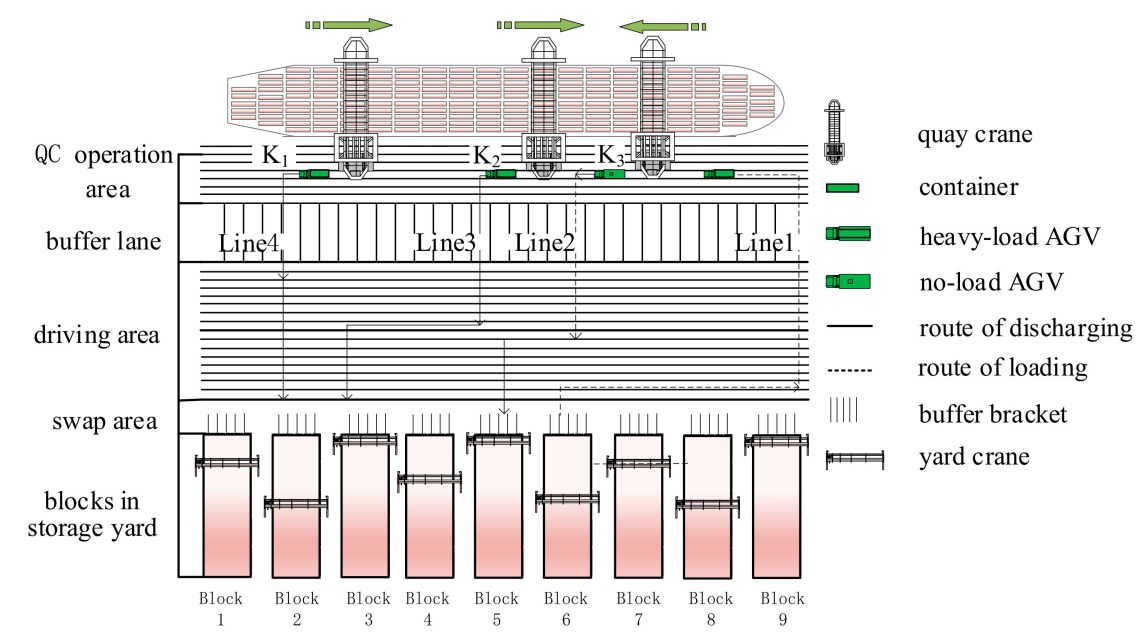

Figure 1. Automated container terminal layout and (AGV) transportation process. 
In this study, the joint configuration and the scheduling problem of the dual-trolley quay crane and an AGV in a single-ship operation mode are divided into two phases. In the first phase, the dual-trolley quay cranes are configured according to the number and location of all containers. A task is defined as a set of containers to be discharged (loaded) from (at) either the hold or the deck of the same ship bay. The center of gravity (CG) of ship must be kept within a certain range to meet the stability requirements. The precedence of the containers is that the discharged container in the hold is after the container on the deck, and the container on the deck is loaded after the container in the hold. Container numbering in each bay is based on the precedence relationships between containers. Figure 2 presents an example for a ship handling loading and discharging containers in six bays. The tasks are indexed sequentially from discharging containers to loading (bay 1 to bay 6). For example, the discharged 32 containers in bay 1 is indexed task 1 , and the total number of loading and discharging tasks is 24 .

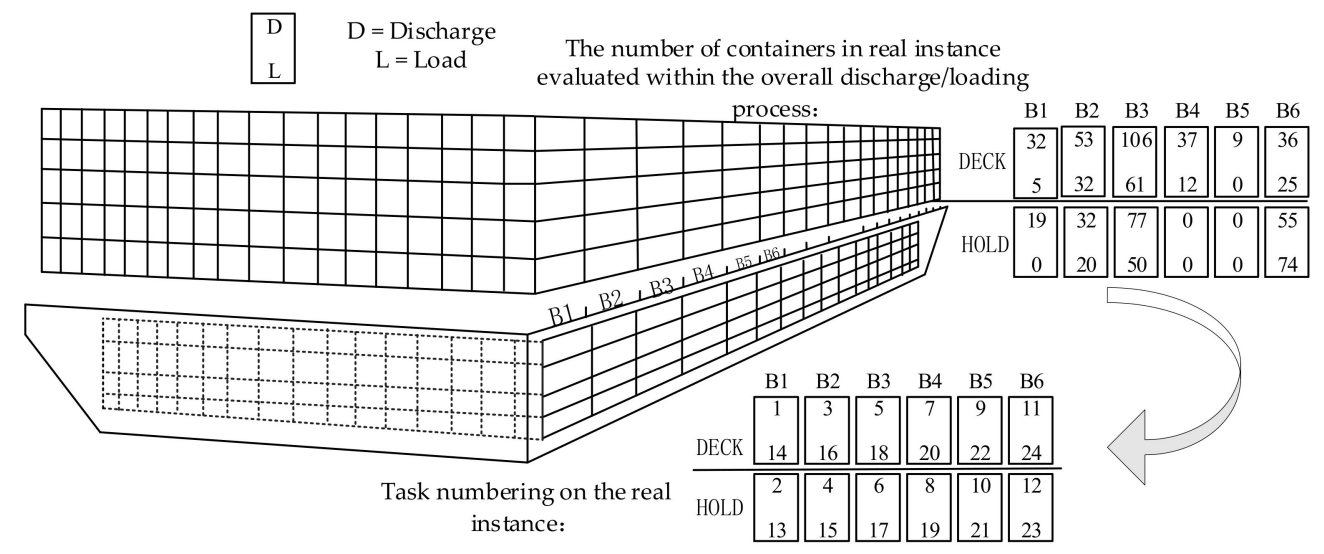

Figure 2. Example of containers and tasks on a ship.

In the second phase, the containers that differ in location need to be distributed from an AGV's point of view. One container is a unit during the dispatching process of an AGV. As shown in Figure 1, after picking up the loaded containers in block 6, the AGV goes to quay crane K3 to deliver the containers to the ship bay, which the containers belong to. After the loaded containers are released, the AGV goes to pick up the next loading task in a block of storage areas (Line 2) or operate the next discharging task under the quay crane K2 (Line 3) or quay crane K1 (Line 4). The AGV stops its operating cycle to charge when it has a low supply of electricity. A container with a given index must be handled before all containers with higher indexes located at the same bay. Since the high losses are caused by the delay of the quay crane, it is necessary to ensure that the main trolley does not wait for the AGV during handling.

This study is based on the following assumptions: (1) All containers are of the same type, and all dual-trolley quay cranes and AGVs have the same performance and fuel consumption, because only one container $(20 \mathrm{ft}$ or $40 \mathrm{ft}$ ) can be handled at the same time by the QC or the AGV. (2) The longitudinal stability of a container ship is considered. (3) Traffic congestion and path conflict of AGVs are not considered. (4) Buffer size for blocks and QC is considered as given and all automated rail mounted gantry cranes (ARMGs) have the same performance and fuel consumption.

\subsection{Phase I: Mathematical Formulation for Configuration and Scheduling of a Dual-Trolley Quay Crane}

All the sets, parameters, and decision variables of the phase I are shown in Table 1. 
Table 1. Parameters of the phase I.

\begin{tabular}{|c|c|c|}
\hline \multicolumn{3}{|l|}{ Sets } \\
\hline$I$ & \multicolumn{2}{|c|}{$\begin{array}{l}\text { represents the set of all tasks, indexed by } i \text {, where } i \text { is a loading/discharging } \\
\text { operation of the same bay on the deck or hold }\end{array}$} \\
\hline$N$ & \multicolumn{2}{|c|}{ represents the set of all containers, indexed by $n$} \\
\hline K & \multicolumn{2}{|c|}{ represents the set of all QCs, indexed by $k$} \\
\hline$T$ & \multicolumn{2}{|c|}{ represents the set of time units, indexed by $t$} \\
\hline \multicolumn{3}{|c|}{ Parameters } \\
\hline$L_{\text {safe }}$ & \multicolumn{2}{|c|}{ safety margin between two consecutive cranes (in number of bays) } \\
\hline$t_{f}$ & \multicolumn{2}{|c|}{ available laytime of the ship } \\
\hline $\bar{G}_{i}^{0}$ & \multicolumn{2}{|c|}{ center gravity of the ship before starting the operations } \\
\hline$G_{i}^{t}$ & \multicolumn{2}{|c|}{ workload before starting the operations in task $i$} \\
\hline & \multicolumn{2}{|c|}{ handled workload at time segment $t$ in task $i$} \\
\hline shift & \multicolumn{2}{|c|}{ maximum allowable shift with the center gravity of the ship (in number of bays) } \\
\hline$\delta$ & \multicolumn{2}{|c|}{1 for loading operations; -1 for discharging operations } \\
\hline$\rho_{i}^{t}$ & \multicolumn{2}{|c|}{ weight of task $i$ at time segment $t$} \\
\hline$\tau_{1}$ & \multicolumn{2}{|c|}{ the time required for QCs to move a bay in the direction of the ship } \\
\hline$\tau_{2}$ & \multicolumn{2}{|c|}{ the average time required for the QCs to pick up and release a container } \\
\hline$c_{1}$ & \multicolumn{2}{|c|}{ unit time energy consumption for QC loading and discharging } \\
\hline$c_{2}$ & \multicolumn{2}{|c|}{ unit time energy consumption for QC moving } \\
\hline$c_{3}$ & \multicolumn{2}{|c|}{ unit energy consumption for QC waiting } \\
\hline$N_{i}$ & \multicolumn{2}{|c|}{ workload in number of containers at tasks $i$} \\
\hline$l_{i}$ & \multicolumn{2}{|c|}{ location of task (in ship-bay number) } \\
\hline$w_{i j k}$ & \multicolumn{2}{|c|}{$\begin{array}{l}\text { the time that the QC } k \text { needs to waiting before starting the task } j \text { after the } \\
\text { completed task } i\end{array}$} \\
\hline \multicolumn{3}{|c|}{ Decision variables } \\
\hline \multirow{2}{*}{$x_{i k}^{t}$} & 1 & if $Q C k$ performs task $i$ at time segment $t$ \\
\hline & 0 & Otherwise \\
\hline \multirow{2}{*}{$x_{i j k}$} & 1 & If QC $k$ performs task $j$ after performing task $i$ \\
\hline & 0 & Otherwise \\
\hline
\end{tabular}

Object 1: Minimize the total energy consumption of QCs which includes working energy consumption, moving energy consumption, and waiting energy consumption.

$$
\min f_{1}=C_{1} \times \sum_{t=1}^{T} \sum_{i=1}^{I} \sum_{k=1}^{K} x_{i k}^{t} N_{i} \tau_{2}+C_{2} \times \sum_{i=1}^{I} \sum_{j=1}^{I} \sum_{k=1}^{K}\left|l_{j}-l_{i}\right| \times x_{i j k} \tau_{1}+C_{3} \times \sum_{i=1}^{I} \sum_{j=1}^{I} \sum_{k=1}^{K} x_{i j k} w_{i j k}
$$

s.t.

$$
\begin{gathered}
\sum_{k=1}^{K} x_{i k}^{t}=1 \forall i \in I, t \in T \\
\sum_{t=1}^{T} \sum_{k=1}^{K} x_{i k}^{t}=N_{i} \forall i \in I \\
\sum_{j=1}^{I} x_{i j k}-\sum_{j=1}^{I} x_{j i k}=0 \forall i \in I, \forall k \in K \\
\sum_{t=1}^{T} \sum_{i=1}^{I} \sum_{k=1}^{K} x_{i k}^{t} N_{i} \tau_{2}+\sum_{k=1}^{K} \sum_{j=1}^{I} \sum_{i=1}^{I}\left|l_{j}-l_{i}\right| \times x_{i j k} \tau_{1}+\sum_{k=1}^{K} \sum_{j=1}^{I} \sum_{i=1}^{I} x_{i j k} w_{i j k} \leq t_{f} \forall i, j \in I, \forall k \in K \\
\sum_{i=1}^{I} x_{i k}^{t} l_{i}-\sum_{j=1}^{I} x_{j k^{\prime}}^{t} l_{j} \geq L_{s a f e} \forall k, k^{\prime} \in K, t \in T
\end{gathered}
$$




$$
\begin{gathered}
g_{0}-\text { shift } \leq \frac{\sum_{i=1}^{I}\left(\delta G_{i}^{t}+G_{i}^{0}\right) \rho_{i}^{t}\left(l_{i}-\frac{1}{2}\right)}{\sum_{i=1}^{I}\left(\delta G_{i}^{t}+G_{i}^{0}\right) \rho_{i}^{t}} \leq g_{0}+\text { shift } \forall t \in T \\
x_{i k}, x_{i j k} \in\{0,1\} \forall i, j \in I, \forall k \in K
\end{gathered}
$$

Constraint (2) ensures that each task is performed by one QC. Constraint (3) guarantees that all containers workload will be handled. Constraint (4) guarantees that each task has one and only one predecessor task and a dummy task. Evidently, the largest time required to service all tasks cannot exceed the available laytime, which is ensured through constraint (5). Constraint (6) enforces the safety margin between QCs. Constraint (7) ensures that the shift of a ship's center of gravity must be maintained within acceptable limits. Finally, the binary nature of the decision variable is specified by constraint (8).

\subsection{Phase II: Mathematical Formulation for AGV Scheduling}

The handling time of containers by the QC can be obtained through the first phase. In this part, loaded and discharged containers are assigned to each AGV and the sequence of operations is scheduled while ensuring that no quay crane is delayed. All the sets, parameters, and decision variables are shown in Table 2.

\begin{tabular}{|c|c|c|}
\hline \multicolumn{3}{|l|}{ Sets } \\
\hline$L$ & \multicolumn{2}{|c|}{ represents the set of loaded containers } \\
\hline$D$ & \multicolumn{2}{|c|}{ represents the set of discharged containers } \\
\hline C & \multicolumn{2}{|c|}{ represents the set of yard cranes(YCs), indexed by $c$} \\
\hline$A$ & \multicolumn{2}{|c|}{ represents the set of AGVs, indexed by $a$} \\
\hline \multicolumn{3}{|c|}{ Parameters: } \\
\hline$T_{u k}^{q}$ & \multicolumn{2}{|c|}{ planned handling time of the $n$th container by main trolley of quay crane $k$} \\
\hline$T_{n k}^{n k}$ & \multicolumn{2}{|c|}{ planned handling time of the $n$th container by gantry trolley of quay crane $k$} \\
\hline$S_{n c}^{n k}$ & \multicolumn{2}{|c|}{ planned handling time of the $n$th container by the yard crane $c$} \\
\hline$E T_{u k}^{O}$ & \multicolumn{2}{|c|}{ earliest starting time of quay crane $k$ for container $n$} \\
\hline$L T_{n k}^{n}$ & \multicolumn{2}{|c|}{ latest starting time of quay crane $k$ for container $n$} \\
\hline$E T_{n c}^{n k}$ & \multicolumn{2}{|c|}{ earliest starting time of yard crane $c$ for container $n$} \\
\hline$L T_{n \mathcal{G}}^{G}$ & \multicolumn{2}{|c|}{ latest starting time of yard crane $c$ for container $n$} \\
\hline$C_{4}$ & \multicolumn{2}{|c|}{ unit time waiting energy consumption for gantry trolley of QC } \\
\hline$C_{5}$ & \multicolumn{2}{|c|}{ unit time energy consumption for AGV convey containers. } \\
\hline$C_{6}$ & \multicolumn{2}{|c|}{ unit time moving energy consumption for no-load AGV } \\
\hline$C_{7}$ & \multicolumn{2}{|c|}{ unit time waiting energy consumption for AGV } \\
\hline$p_{1}$ & \multicolumn{2}{|c|}{ size of transit platform of on dual-trolley quay crane } \\
\hline$p_{2}$ & \multicolumn{2}{|c|}{ size of buffer bracket in each block } \\
\hline$\tau_{3}$ & \multicolumn{2}{|c|}{ the time required for $\mathrm{QC}$ to pick up/put down a container } \\
\hline$\tau_{4}$ & \multicolumn{2}{|c|}{ the time required for yard crane to pick up/put down a container } \\
\hline$\tau_{5}$ & \multicolumn{2}{|c|}{ the time required for AGV to make a round trip to the charging station } \\
\hline$v_{1}$ & \multicolumn{2}{|c|}{ speed of load AGV } \\
\hline$v_{0}$ & \multicolumn{2}{|c|}{ speed of no-load AGV } \\
\hline$T^{Q}$ & \multicolumn{2}{|c|}{ actual handling time of the $n$th container by main trolley of quay crane $k$} \\
\hline & \multicolumn{2}{|c|}{ actual handling time of the $n$th container by gantry trolley of quay crane $k$} \\
\hline \multicolumn{3}{|c|}{$\begin{array}{c}n k \\
\text { Decision variables }\end{array}$} \\
\hline \multirow{2}{*}{$x_{n a}$} & 1 & if container $n$ is assigned by the $a$ th AGV. \\
\hline & 0 & Otherwise \\
\hline \multirow{2}{*}{$x_{n n \prime a}$} & 1 & if $a$ th AGV performs container $n^{\prime}$ after performing container $n$ \\
\hline & 0 & Otherwise \\
\hline \multirow{3}{*}{$u_{n a}$} & & if $a$ th AGV's power is less than the safe power after \\
\hline & & performing container $n$ \\
\hline & 0 & Otherwise \\
\hline
\end{tabular}

Table 2. Parameters of the phase II. 
The objective is to minimize the energy consumption of the schedule in order to increase the utilization rate of AGVs.

$$
\begin{gathered}
f_{2}=C_{4} \times \sum_{k=1}^{K} \sum_{n=1}^{N}\left(T_{n k}^{E}-T_{n k}^{e}\right)+C_{5} \times \sum_{a=1}^{A} \sum_{n=1}^{N} x_{n a} t_{n a}+C_{6} \times \sum_{a=1}^{A} \sum_{n^{\prime}=1}^{N} \sum_{n=1}^{N} x_{n n^{\prime} a} t_{n n^{\prime} a} \\
+C_{7} \times\left(\sum_{a=1}^{A} \sum_{c=1}^{C} \sum_{n=1}^{N} w_{n c a}+\sum_{a=1}^{A} \sum_{k=1}^{K} \sum_{n=1}^{N} w_{n k a}\right)
\end{gathered}
$$

s.t.

$$
\begin{aligned}
& \sum_{a=1}^{A} x_{n a}=1 \quad \forall n \in N \\
& \sum_{n=1}^{N} x_{0 n k}=1 \quad \forall k \in K \\
& \sum_{n=1}^{N} x_{n \omega k}=1 \quad \forall k \in K \\
& T_{n k}^{Q} \leq T_{n k}^{q} \quad \forall n \in N \\
& E T_{n k}^{O}=T_{n k}^{q}-p_{1} \times \tau_{2}, L T_{n k}^{O}=T_{n k}^{q} \quad \forall n \in L, \forall k \in K \\
& E T_{n k}^{O}=T_{n k^{\prime}}^{q} L T_{n k}^{O}=T_{n k}^{q}+p_{1} \times \tau_{2} \quad \forall n \in D, \forall k \in K \\
& E T_{n c}^{O} \leq T_{n k}^{E}+\frac{1}{2} \tau_{3} \leq L T_{n c}^{O} \quad \forall n \in L, \forall k \in K \\
& E T_{n c}^{O} \leq T_{n k}^{E}-\frac{1}{2} \tau_{3} \leq L T_{n c}^{O} \quad \forall n \in D, \forall k \in K \\
& T_{n k}^{E}=\max \left\{T_{n a}, T_{n k}^{e}\right\} \quad \forall n \in D, \forall k \in K, \forall a \in A \\
& T_{n k}^{E}=\max \left\{T_{n a}+t_{n a}, T_{n k}^{e}\right\} \quad \forall n \in L, \forall k \in K, \forall a \in A \\
& T_{n^{\prime} k}^{e}=T_{n k}^{E}+\tau_{3} \quad \forall n, n^{\prime} \in N, \forall k \in K \\
& E T_{n c}^{G}=S_{n c}, L T_{n c}^{G}=S_{n c}+p_{2} \times \tau_{4} \quad \forall n \in L, \forall c \in C \\
& E T_{n c}^{G}=S_{n c}-p_{2} \times \tau_{4}, L T_{n c}^{G}=S_{n c} \quad \forall n \in D, \forall c \in C \\
& E T_{n c}^{G} \leq T_{n a} \leq L T_{n c}^{G} \quad \forall n \in L, \forall a \in A, \forall c \in C \\
& E T_{n c}^{G} \leq T_{n a}+t_{n a} \leq L T_{n c}^{G} \quad \forall n \in D, \forall a \in A, \forall c \in C \\
& w_{n k a}=\max \left\{T_{n k}^{e}-T_{n a}-t_{n a}, 0\right\} \quad \forall n \in L, \forall k \in K, \forall a \in A \\
& w_{n k a}=\max \left\{T_{n k}^{e}-T_{n a}, 0\right\} \quad \forall n \in D, \forall k \in K, \forall a \in A \\
& w_{n c a}=\max \left\{E T_{n c}^{G}-T_{n a}, 0\right\} \quad \forall n \in L, \forall a \in A, \forall c \in C \\
& w_{n c a}=\max \left\{E T_{n c}^{G}-T_{n a}-t_{n a}, 0\right\} \quad \forall n \in D, \forall a \in A, \forall c \in C \\
& T_{n a}+t_{n a}+w_{n k a}+t_{n n^{\prime} a}+w_{n^{\prime} c a}+\tau_{5} \times u_{n a} \leq T_{n^{\prime} a}+M\left(1-x_{n n^{\prime} a}\right) \quad \forall n \in L, n^{\prime} \in L \\
& T_{n a}+t_{n a}+w_{n k a}+t_{n m a}+w_{n^{\prime} k a}+\tau_{5} \times u_{n a} \leq T_{n^{\prime} a}+M\left(1-x_{n n^{\prime} a}\right) \quad \forall n \in L, n^{\prime} \in D \\
& T_{n a}+t_{n a}+w_{n c a}+t_{n n^{\prime} a}+w_{n^{\prime} k a}+\tau_{5} \times u_{n a} \leq T_{n^{\prime} a}+M\left(1-x_{n n^{\prime} a}\right) \quad \forall n \in D, n^{\prime} \in L \\
& T_{n a}+t_{n a}+w_{n c a}+t_{n n^{\prime} a}+w_{n^{\prime} k a}+\tau_{5} \times u_{n a} \leq T_{n^{\prime} a}+M\left(1-x_{n n^{\prime} a}\right) \quad \forall n \in D, n^{\prime} \in D
\end{aligned}
$$




$$
\begin{aligned}
& \mu=\frac{C_{5} \times \sum_{a=1}^{A} \sum_{n=1}^{N} x_{n a} t_{n a}}{C_{5} \times \sum_{a=1}^{A} \sum_{n=1}^{N} x_{n a} t_{n a}+C_{6} \times \sum_{a=1}^{A} \sum_{n^{\prime}=1}^{N} \sum_{n=1}^{N} x_{n n^{\prime} a} t_{n n^{\prime} a}+C_{7} \times\left(\sum_{a=1}^{A} \sum_{c=1}^{C} \sum_{n=1}^{N} w_{n c a}+\sum_{a=1}^{A} \sum_{k=1}^{K} \sum_{n=1}^{N} w_{n k a}\right)} \\
& x_{n a}, x_{n n^{\prime} a}, u_{n a} \in\{0,1\} \forall n, n^{\prime} \in N, \forall a \in A \\
& T_{n a} \geq 0 \forall n \in N, \forall a \in A
\end{aligned}
$$

Constraint (10) ensures that each container is served by one AGV. Constraints (11) and (12) imply that each AGV has a starting point and destination. Constraint (13) enforces that the main trolley of the quay crane is not delayed. The time window is determined in constraints (14) and (15) by each QC according to the first phase results. Constraints (16) and (17) ensure that each container is served by quay crane under the time windows. The actual handling time of the $n$th container by quay crane is determined in constraints (18) and (19). Constraint (20) specifies the updated plan handling time of the gantry trolley of the quay crane. The actual handling time of the $n$th container by the yard crane is determined in constraints (21) and (22). Constraints (23) and (24) ensure that each container is served by a yard crane under the time windows. Constraints (25) to (28) imply the waiting time when the AGV arrives early. Constraints (29) to (32) determine the starting time of the next container by one AGV. The utilization is calculated by Constraint (33). Constraints (34) and (35) restrict the domains of variables.

\section{Algorithms}

In this section, we present two algorithms for the configuration and scheduling optimization model of the quay crane and AGV.

\subsection{Enumeration Algorithm for Solving the First Phase Model}

Similar to quay crane scheduling with the non-interference constraints problem (QCSNIP) [25] and the three-dimensional knapsack problem with balancing constraints [26], all tasks must be handled during the laytime and the center of ship must lie into a predefined bayed domain inside the ship. Moreover, Lee [25] proved that the QCSNIP is NP-complete and that no polynomial time algorithm for the exact solution exists. Therefore, in this case, the enumeration algorithm (EA) is proposed for solving the quay crane configuration and scheduling problem with laytime, precedence, safety margin, and stability constraints as described in following steps:

(1) Generation of task assignment matrices of each quay crane

Arrange all tasks according to the precedence as follows: Tasks with a smaller ship bay number are placed first when two tasks of different bays are served by the same quay crane; the task of an on-deck container is earlier than an in-hold container when two discharging tasks of the same bay are served by the same quay crane, and vice versa. The enumeration algorithm is employed to generate the initial task assignment matrices of each quay crane.

To keep the ship stable, the first quay crane moves from the bow to the stern, and the last quay crane moves from the stern to the bow. As shown in Figure 3, QC 1 moves from left to right, and bay 6 belongs to the task assignment matrices of QC 1. QC 2 moves from right to left, and bay 7 belongs to the QC 2's work area. In order to reduce the waiting time of the quay crane at the conflict point, the avoidance strategy is adopted, which means that QC 1 can handle bay 6 in advance, and then return to bay 5 . 


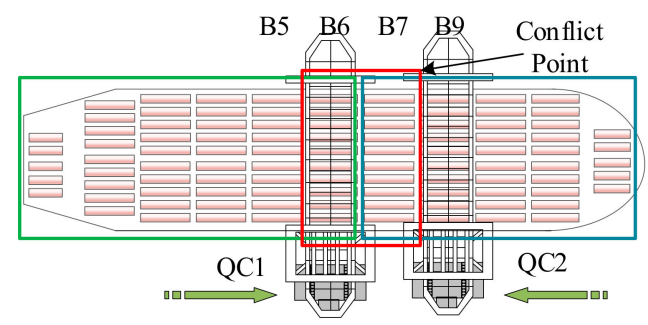

Figure 3. Example of a possible conflict point.

(2) Traversing the set of feasible solutions for the optimal scheduling scheme.

We use a constrained partial enumeration strategy to remove the infeasible solution. Calculating the makespan and stability of the vessel, distance between quay cranes over time, and only keeping schemes that meet the constraints (5) to (7). The scheduling scheme with the minimum objective value are retained.

(3) Determining the optimal configuration scheme.

The optimal configuration was obtained by comparing the values of objectives under the different number of quay cranes configured.

\subsection{Extended Genetic Algorithm for Solving the Second Phase Model}

Since it is very complex and time consuming to explore the whole solution space, we extended the genetic algorithm to solve the second phase model. The genetic algorithm has the advantage of flexibility and imposes no requirement for a problem to be formulated as any particular type. Thus, they can be applied to non-liner problems for which there is a way to encode the quality of a solution. Here, we use an integer chromosomal representation. The algorithm consists of five parts as follows:

\section{(1) Chromosome representation}

A chromosome is represented by a matrix with $2 \times \mathrm{n}$ genes. The gene value in the first line of chromosome represents the number of containers, and the gene value in the second line represents the number of AGVs assigned to each container. According to the stowage plan and the distribution results of the quay crane in the first phase, the number of quay crane and yard crane for each container can be obtained. Figure 4 illustrates an example of a chromosome with 3 QCs, 4 AGVs and 15 containers. The container 27 is operated by yard crane 7 and quay crane 2, assigned to AGV 1 for transportation.

\begin{tabular}{c|c|c|c|c|c|c|c|c|c|c|c|c|c|c|c|} 
Container index & 27 & 50 & 26 & 43 & 19 & 44 & 15 & 1 & 36 & 23 & 2 & 4 & 18 & 24 & 13 \\
\cline { 2 - 29 } & 1 & 1 & 1 & 1 & 2 & 2 & 2 & 3 & 3 & 3 & 4 & 4 & 4 & 4 & 4 \\
\hline QC index & 2 & 3 & 2 & 2 & 1 & 3 & 3 & 1 & 3 & 2 & 2 & 1 & 3 & 1 & 1 \\
YC index & 7 & 5 & 8 & 9 & 6 & 6 & 4 & 7 & 12 & 7 & 10 & 7 & 11 & 12 & 4
\end{tabular}

Figure 4. The chromosome.

(2) Generating initial population

The initial population can be generated randomly or heuristically. Random generation gives the initial population great diversity. A population of individuals is initialized by randomly selecting tasks with the constraints (10) to (12) to make sure every solution is feasible.

(3) Fitness evaluation and chromosomal selection

The chromosomes with high fitness are selected and proceed to the next iteration. Figure 5 shows the procedure to calculate the objective value. After the generation of a population, its fitness value 
should be higher. Hence, the reciprocal value of the objective function is taken as the fitness value and chromosomes with the higher fitness values have a greater chance to survive.

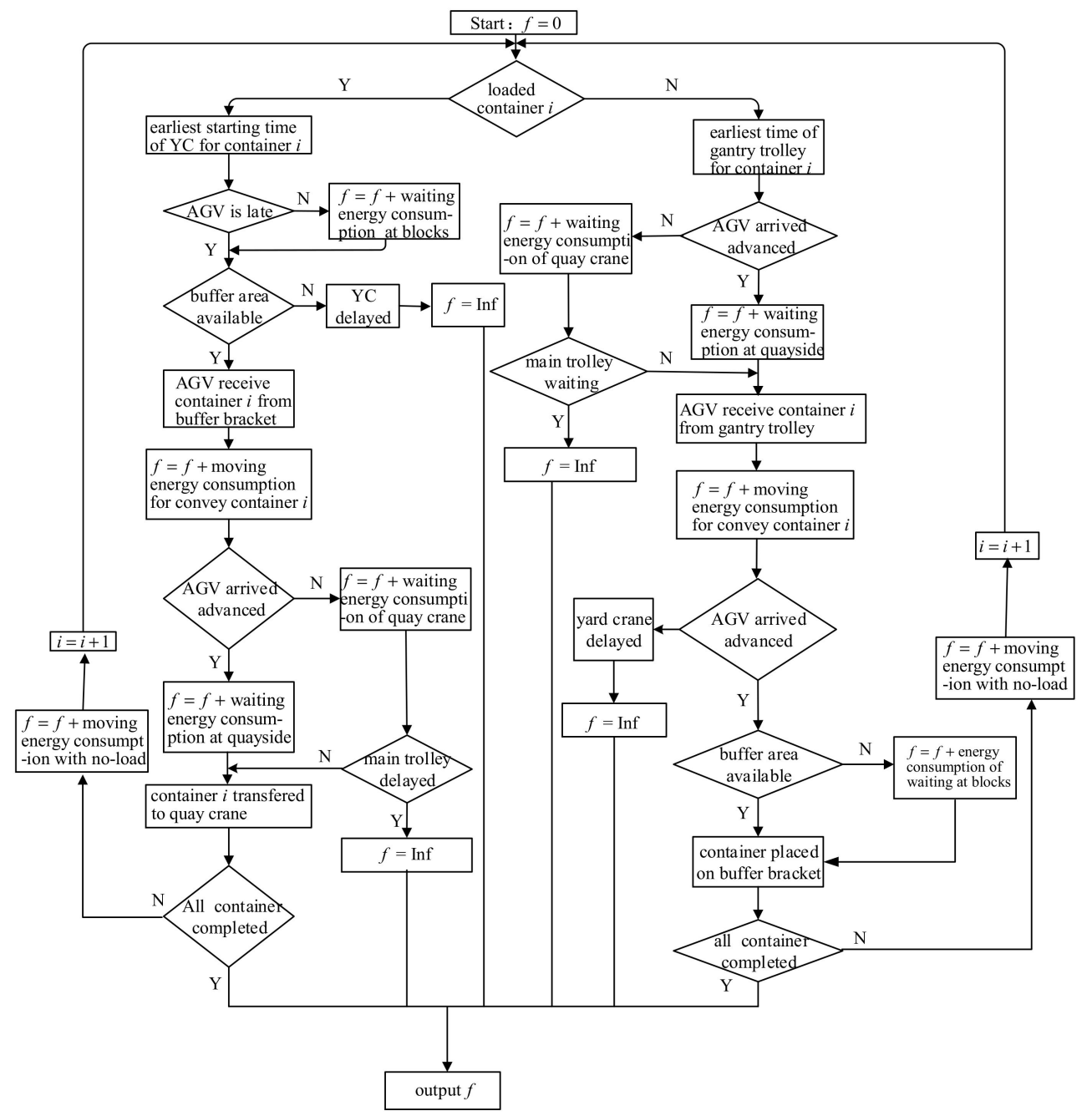

Figure 5. Flowchart for solving the model.

(4) Crossover and mutation

Grouping the chromosomes (individuals) of a population into groups of eight. The parent chromosomes taken from the groups with the highest value are retained for the next generation. Figure 6 presents the produced offsprings from two parent chromosomes. A two-point crossover is used to produce new chromosomes in which the workload of each containers is fully shared between AGVs. The position of the intersection point is randomly generated. The new offsprings are depicted in Figure 6a. These crossovers are applied to generate two cross children per chromosome.

The mutation is achieved by varying the index or number of containers served by each AGV and varying the index of AGVs to containers. We adopt three common types of mutation referred to as swap and insert. The specific changes of the mutation operation are shown in Figure $6 \mathrm{~b}-\mathrm{d}$. These mutations are applied to parent chromosomes generating three mutated children chromosomes. 


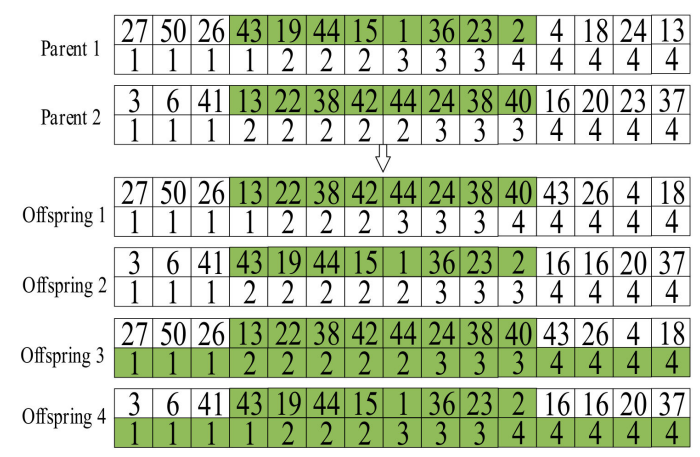

(a) Crossover

Parent \begin{tabular}{c|c|c|c|c|c|c|c|c|c|c|c|c|c|c|}
\hline 27 & 50 & 26 & 43 & 19 & 44 & 15 & 1 & 36 & 23 & 2 & 4 & 18 & 24 & 13 \\
\hline 1 & 1 & 1 & 1 & 2 & 2 & 2 & 3 & 3 & 3 & 4 & 4 & 4 & 4 & 4 \\
\hline
\end{tabular}

Offspring \begin{tabular}{|c|c|c|c|c|c|c|c|c|c|c|c|c|c|c|}
\hline 27 & 50 & 26 & 2 & 19 & 44 & 15 & 1 & 36 & 23 & 43 & 4 & 18 & 24 & 13 \\
\hline 1 & 1 & 1 & 1 & 2 & 2 & 2 & 3 & 3 & 3 & 4 & 4 & 4 & 4 & 4 \\
\hline
\end{tabular}

(b) Mutation 1

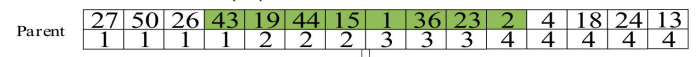
\begin{tabular}{|c|c|c|c|c|c|c|c|c|c|c|c|c|c|c|}
\hline 27 & 50 & 26 & 2 & 23 & 36 & 1 & 15 & 44 & 19 & 43 & 4 & 18 & 24 & 13 \\
\hline 1 & 1 & 1 & 1 & 2 & 2 & 2 & 3 & 3 & 3 & 4 & 4 & 4 & 4 & 4 \\
\hline
\end{tabular}

(c) Mutation2

\begin{tabular}{c|c|c|c|c|c|c|c|c|c|c|c|c|c|c|c|} 
Parent & 27 & 50 & 26 & 43 & 19 & 44 & 15 & 1 & 36 & 23 & 2 & 4 & 18 & 24 & 13 \\
\cline { 2 - 11 } & 1 & 1 & 1 & 1 & 2 & 2 & 2 & 3 & 3 & 3 & 4 & 4 & 4 & 4 & 4 \\
\hline
\end{tabular} \begin{tabular}{|c|c|c|c|c|c|c|c|c|c|c|c|c|c|c|}
\hline 27 & 50 & 26 & 43 & 19 & 44 & 15 & 1 & 36 & 23 & 2 & 4 & 18 & 24 & 13 \\
\hline 1 & 1 & 2 & 2 & 2 & 2 & 3 & 3 & 3 & 3 & 3 & 3 & 4 & 4 & 4 \\
\hline
\end{tabular}

(d) Mutation3

Figure 6. Crossover and mutation.

(5) Stopping criterion

The GA algorithm terminates when the algorithm reaches the maximum number of iterations.

\section{Case Studies}

\subsection{Experimental Setting}

The numerical tests in this section are conducted on a server Intel (R) Core(TM) i7-7700 CPU@ 3.6GHz with the memory of 16.00GB. The real data comes from Shanghai Yangshan Phase IV automated container terminal located in China, which has high throughput. The layout of the terminal and the traffic rules for horizontal transportation are shown in Figure 1. The positional distribution of containers on the ship presented in [13] is shown in Figure 7, and there are 20 bays waiting for loading or discharging. The configuration of dual-trolley quay crane + AGV + automated rail mounted gantry crane (ARMG) is adopted in the research, and some of relevant parameters of quay crane and AGV, such as travelling speed and operating energy consumption $[27,28]$, are shown in Table 3.

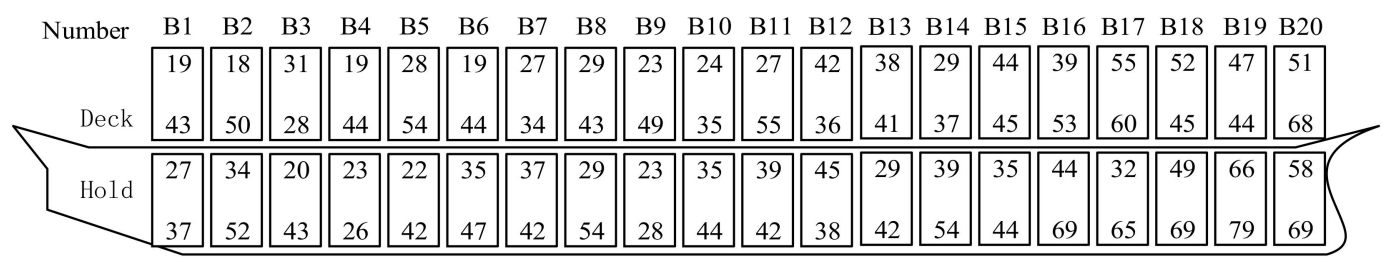

Figure 7. Number of loaded and discharged containers of ship. 
Table 3. Device-related parameter values.

\begin{tabular}{|c|c|c|c|}
\hline Parameters & Value & Parameters & Value \\
\hline$\tau_{1} / \min$ & 1 & $\mathrm{C}_{1} /[\mathrm{kw} \cdot \mathrm{h}]$ & 91.24 \\
\hline$\tau_{2} / \min$ & 2 & $C_{2} /[\mathrm{kw} \cdot \mathrm{h}]$ & 70.18 \\
\hline$\tau_{3} / \min$ & 1 & $\mathrm{C}_{3} /[\mathrm{kw} \cdot \mathrm{h}]$ & 49.6 \\
\hline$\tau_{4} / \min$ & 3 & $\mathrm{C}_{4} /[\mathrm{kw} \cdot \mathrm{h}]$ & 49.6 \\
\hline$\tau_{5} / \min$ & 5 & $\begin{array}{c}\mathrm{C}_{5} /[\mathrm{kw} \cdot \mathrm{h} . \\
\left.\mathrm{vch}^{-1}\right]\end{array}$ & 21 \\
\hline$v_{1} /\left[\mathrm{m} \cdot \mathrm{min}^{-1}\right]$ & 210 & $\begin{array}{c}\mathrm{C}_{6} /[\mathrm{kw} \cdot \mathrm{h} . \\
\left.\mathrm{vch}^{-1}\right]\end{array}$ & 14 \\
\hline$v_{0} /\left[\mathrm{m} \cdot \min ^{-1}\right]$ & 350 & $\begin{array}{c}C_{7} /[\mathrm{kw} \cdot \mathrm{h} \cdot \\
\left.\mathrm{vch}^{-1}\right]\end{array}$ & 9 \\
\hline
\end{tabular}

There are nine blocks in the container yard for stacking containers waiting to be discharged and loaded. The capacity of the transfer platform of dual-trolley quay crane is set to two containers, and the safety margin between two quay cranes is set to one bay. The capacity of buffer bracket of each block is set to five containers. An AGV can last 480 minutes after the battery is replaced.

\subsection{Optimization Results}

\subsubsection{The Results of Quay Crane Scheduling and Configuration in the First Phase}

The calculation process is coded in Matlab2016. In all instances, the permitted shift of the vessel's CG is assumed to be two bay lengths. The laytime of the ship is assumed to be $44 \mathrm{~h}$. In this test, two quay cranes cannot help to complete within $44 \mathrm{~h}$. When the number of cranes is 3 or 4 , the makspan was below the laytime limit, however, the energy consumption and time consumption of the latter was higher than the former for the reason that waiting energy consumption increased. Therefore, the optimal number of quay cranes is three, the minimum energy consumption of quay cranes is 10,024 $\mathrm{kw} \cdot \mathrm{h}$, and the makespan is $2577 \mathrm{~min}$. The optimal scheduling scheme is as follows: For the discharging operation, $\mathrm{QC}_{1}$ is responsible for bays 1 to $6, \mathrm{QC}_{2}$ is responsible for bays 7 to 16 , and $\mathrm{QC}_{3}$ discharges bays 17 to 20; for the loading operation, $\mathrm{QC}_{1}$ is responsible for bays 1 to $10, \mathrm{QC}_{2}$ is responsible for bays 11 to 16 , and $\mathrm{QC}_{3}$ loads bays 17 to 20 . Figure 8 reports the obtained results. $\mathrm{QC}_{1}$ finished discharging operation and started loading operation at the 599th $\mathrm{min}$, and $\mathrm{QC}_{3}$ finished discharging operation at the 1369th min. The synchronous operation time was $770 \mathrm{~min}$, which was conducive to reducing the AGV's no-load time. A safety margin between two consecutive cranes is maintained at any time with no intersection of the moving routes.

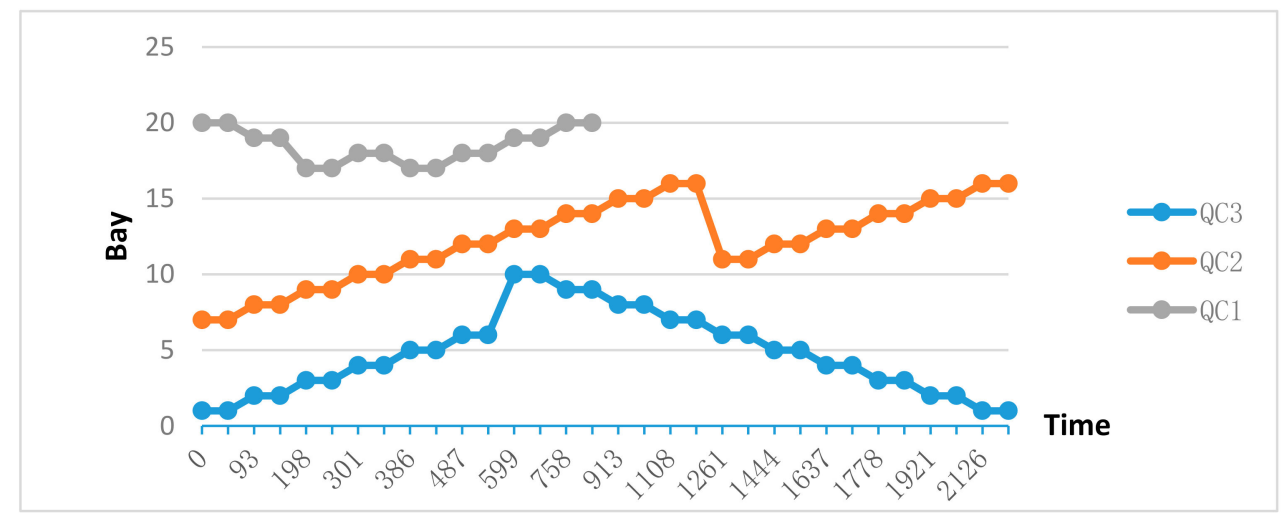

Figure 8. Path to the dual-trolley quay cranes. 


\subsubsection{The Results of AGV Scheduling and Configuration in the Second Phase}

In the second phase, 3769 containers are considered. On the basis of the results of first phase, coding genetic algorithm framework with MATLAB is used to obtain the AGV scheduling schemes. On the basis of the preliminary tests, the population size, the probability of crossover, the probability of mutation, and the maximum number of generations are set to $120,0.8,0.2$, and 1500, respectively. The total energy consumption of seven AGVs is $3202.4 \mathrm{kw} \cdot h$, and the utilization rate of the AGVs is $55.5 \%$. The scheduling schemes of the AGVs are shown in Figure $9 \mathrm{a}-\mathrm{g}$.

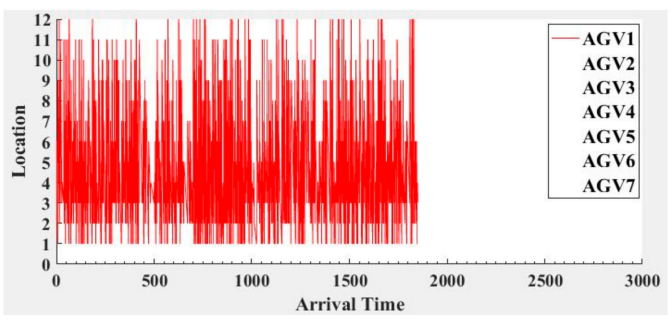

(a) Travel route of AGV1

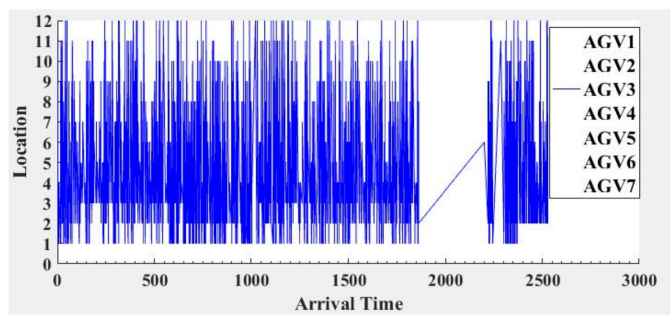

(c) Travel route of AGV3

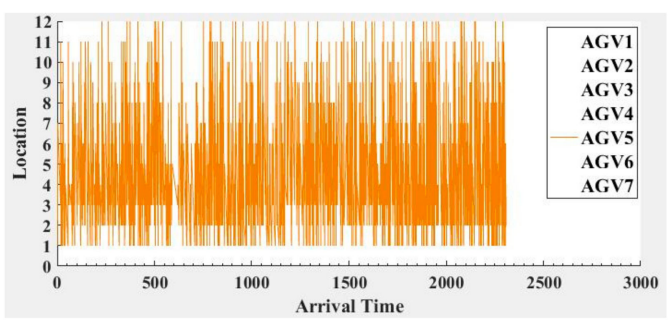

(e) Travel route of AGV5

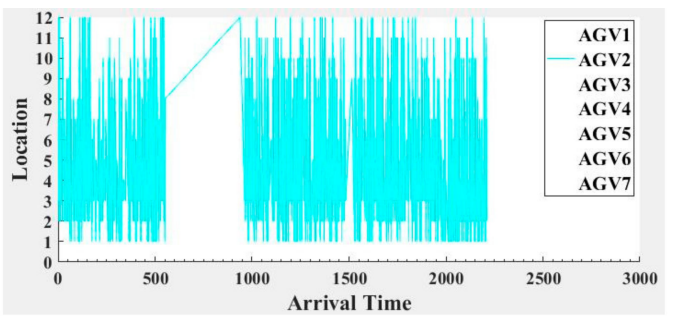

(b) Travel route of AGV2

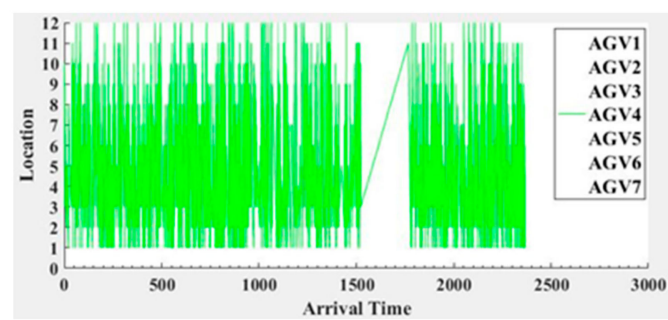

(d) Travel route of AGV4

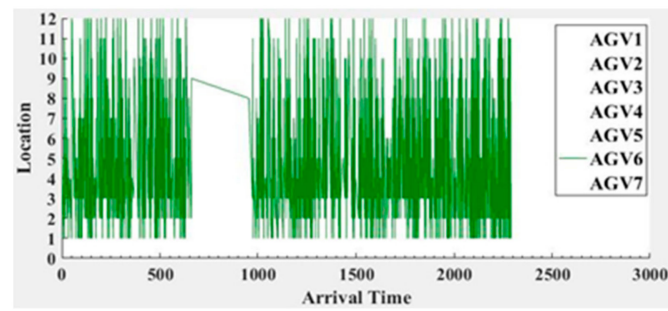

(f) Travel route of AGV6

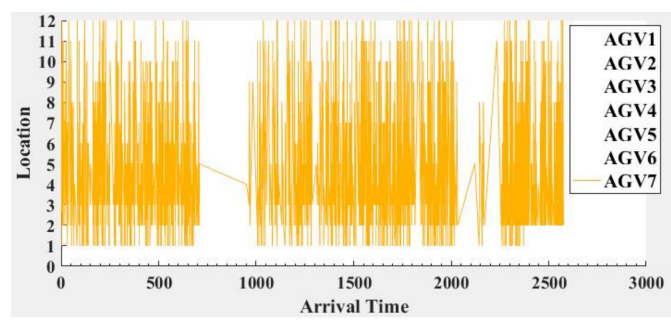

(g) Travel route of AGV7

Figure 9. The travel route of AGVs.

In order to verify the effectiveness of the model and algorithm, we illustrate the comparison of these strategies, in terms of laytime of ships and configuration principles of an AGV. The comparison is shown in Table 4 where principle 1 is minimum energy consumption, principle 2 is minimum number of AGV configured, and principle 3 is set configure ratio of quay cranes and AGVs with 1:3 mentioned in [29]. We can see that the shorter the laytime is the more quay cranes need to be configured. For the same number of QCs, the energy consumption increased when the laytime decreased. 
Table 4. The results of different laytime and different AGV configuration principles.

\begin{tabular}{|c|c|c|c|c|c|c|c|c|c|c|c|}
\hline \multirow{2}{*}{ Text } & \multirow{2}{*}{$t_{f} / h$} & \multirow{2}{*}{$\mathbf{T}_{\mathbf{q}_{\max }} / \mathbf{h}$} & \multirow{2}{*}{$\begin{array}{c}\mathrm{K} / \\
\text { piece }\end{array}$} & \multirow{2}{*}{$\mathbf{f}_{1} / \mathbf{k w} \cdot \mathbf{h}$} & \multirow{2}{*}{$\begin{array}{c}\min \left(\mathbf{f}_{1}+\mathbf{f}_{2}\right) \\
/ \mathbf{k w} \cdot \mathbf{h}\end{array}$} & \multicolumn{2}{|c|}{ Principle 1} & \multicolumn{2}{|c|}{ Principle 2} & \multicolumn{2}{|c|}{ Principle 3} \\
\hline & & & & & & V/vch & $\mathbf{f}_{2} / \mathbf{k w} \cdot \mathbf{h}$ & V/vch & $\mathbf{f}_{2} / \mathbf{k w} \cdot \mathbf{h}$ & V/vch & $\mathbf{f}_{2} / \mathbf{k w} \cdot \mathbf{h}$ \\
\hline 1 & 48 & 1 & 2 & 1 & 1 & 1 & 1 & 1 & 1 & 1 & 1 \\
\hline 2 & 44 & 42.95 & 3 & $10,024.4$ & 13226.8 & 7 & 3202.4 & 6 & 3269.8 & 9 & 3548.8 \\
\hline 3 & 40 & 38.60 & 3 & $10,058.2$ & 13377.6 & 7 & 3319.4 & 7 & 3319.4 & 9 & 3594.5 \\
\hline 4 & 36 & 34.11 & 4 & $10,180.9$ & 13450.3 & 8 & 3269.4 & 8 & 3269.4 & 12 & 3515.8 \\
\hline 5 & 32 & 31.53 & 4 & $10,180.9$ & 13429.8 & 8 & 3248.9 & 7 & 3414.2 & 12 & 3459.6 \\
\hline
\end{tabular}

Figure 10 depicts the utilization rates of AGVs with different configuration principles. In all tests, the third principle has the lower utilization rate. For example, in test 2 where nine AGVs are assigned to three QCs, the utilization rate is as low as $54 \%$. This indicates that a large number of AGVs cannot decrease energy consumption, and a good planning coordination technique is necessary. The second principle is not competitive because it can result in a high utilization for each AGV and high total energy consumption with quay crane delayed. The first principle saves a significant percentage of the number of AGVs without any delay, under the decoupling effect of the transit platform of dual-trolley quay crane and the buffer bracket at storage yard. For example, in test 5 where seven AGVs are assigned to four QCs the ratio rose to 1:1.75. As compared with principle 3, the first principle has shorter waiting time for AGVs, for example, in test 2 where seven AGVs are assigned to three QCs, the energy consumption is reduced $10.8 \%$.

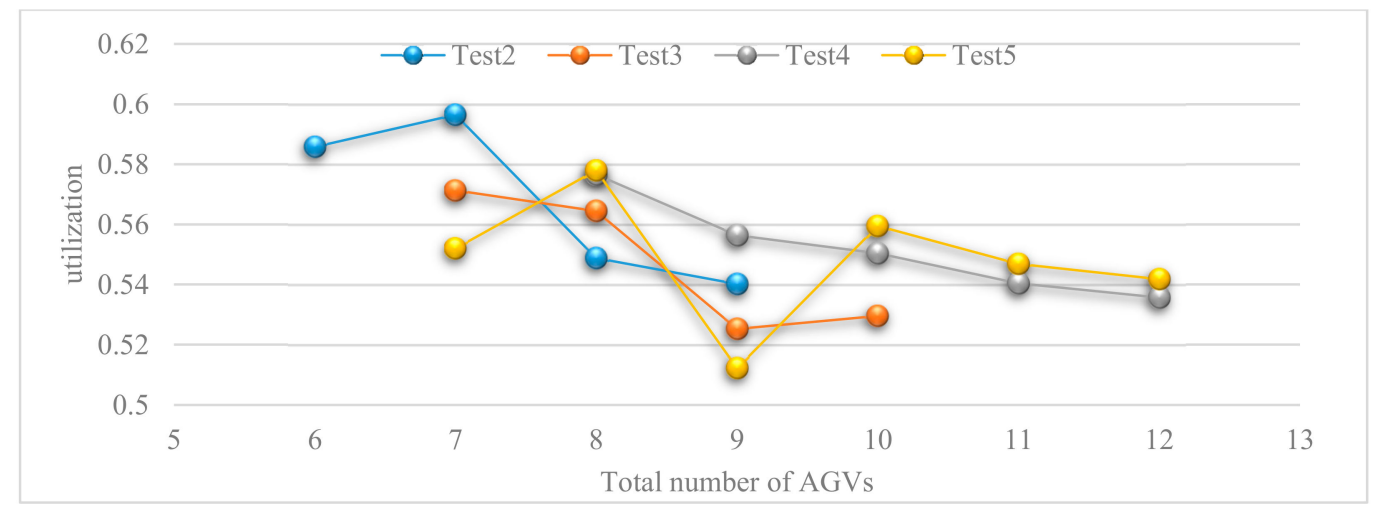

Figure 10. The utilization rate of AGV.

\section{Conclusions}

In this paper, we have discussed a method for joint configuration and scheduling optimization of a dual-trolley quay crane and AGVs. The problem has introduced as a scheduling problem with stability constraints, available laytime constraints, precedence constraints, safety margin constraints, buffer size constraints, and AGVs' endurance time constraints. A two-phase optimization model is established, in which the optimization goal of the first phase is to minimize the energy consumption of the dual-trolley quay crane, and that of the second phase is to minimize the energy consumption and maximize the utilization rate of the AGVs. The EA algorithm is used to calculate the working time of the main trolley for each container. The improved genetic algorithm is used to solve the second-phase model, and the optimal operation sequence of AGV can be obtained without main trolley delay. Furthermore, the effectiveness of the model and algorithm is verified by analyzing and comparing the dual-trolley quay crane and AGV configuration and scheduling schemes under different available laytimes and different AGV configuration principles. The research in this study resulted in the following conclusions:

(1) A two-phase mixed integer programming model is proposed. The enumeration algorithm is developed to solve the quay crane scheduling, and the genetic algorithm is improved to obtain 
the AGV scheduling, in order to complete the loading and discharging operations of all containers on the ship and maintain the stability of the ship in laytime.

(2) The available laytime has an impact on the configured number of quay cranes. The shorter the laytime, the more the quay cranes are configured, and the more total energy consumption of the handling operations. The stability has an impact on the movement sequence of the quay cranes. The order of operations can be changed to reduce the wait time of the quayside and keep the ship stable.

(3) The single ship's operation and scheduling mode has been studied by converting the buffer platform of the dual-trolley quay crane and the buffer bracket of block constraints into time window constraints. According to the results of this experiment, it was found that the ratio of quay cranes and AGVs after optimization is about 1:2, which is higher than the optimal ratio 1:3 obtained by a simulation in the study by [29]. Regardless of the influence of an uncertain environment and other factors, in case 5, the configuration ratio of quay cranes and AGV was 1:1.75, without delay of the main trolley. However, because of the energy consumption caused by the waiting of quay cranes and yard cranes, the energy consumption of the handling operation could not be lowest.

This research is more in line with the actual loading and discharging process at an automated container terminal, but there are still some shortcomings. This paper did not consider the uncertain factors in the loading and discharging process, such as the path conflict and congestion, which affect the handling time of containers in the actual environment. By changing the input value, such as the speed of the AGV or the efficiency of the QC in the model, from deterministic to indeterminate, the problem of quay crane and AGV joint scheduling affected by uncertain factors could be the future research direction.

Author Contributions: Conceptualization, L.Y. and H.F.; methodology, L.Y. and H.F.; software, L.Y.; validation, L.Y. and H.F. case study, L.Y. and H.F.; investigation, L.Y.; resources, H.F.; data curation, L.Y.; writing-original draft preparation, L.Y. and C.Z.; writing—review and editing, H.F.; supervision, H.F.; project administration, H.F. All authors have read and agreed to the published version of the manuscript.

Funding: This research was funded by National Natural Science foundation of China (no. 61473053), the project of Double First-Class Construction (Innovative Projects) (no. CXXM2019SS013).

Conflicts of Interest: The authors declare no conflict of interest.

\section{References}

1. Sim, J. A carbon emission evaluation model for a container terminal. J. Clean. Prod. 2018, 186, 526-533. [CrossRef]

2. He, J.; Huang, Y.; Yan, W. Yard crane scheduling in a container terminal for the trade-off between efficiency and energy consumption. Adv. Eng. Inf. 2015, 29, 59-75. [CrossRef]

3. Chang, D.; He, J.; Bian, Z. An investigation into berth and quay crane scheduling for container terminals based on knowledge. In Proceedings of the 2010 International Conference on Future Information Technology and Management Engineering, Changzhou, China, 9 October 2010; pp. 63-66.

4. He, J. Berth allocation and quay crane assignment in a container terminal for the trade-off between time-saving and energy-saving. Adv. Eng. Inf. 2016, 30, 390-405. [CrossRef]

5. Chang, D.; Fang, T.; Fan, Y. Dynamic rolling strategy for multi-vessel quay crane scheduling. Adv. Eng. Inf. 2017, 34, 60-69. [CrossRef]

6. Kovalyov, M.Y.; Pesch, E.; Ryzhikov, A. A note on scheduling container storage operations of two non-passing stacking cranes. Networks 2018, 71, 271-280. [CrossRef]

7. Liu, D.; Ge, Y.E. Modeling assignment of quay cranes using queueing theory for minimizing $\mathrm{CO}_{2}$ emission at a container terminal. Transp. Res. Part D Transp. Environ. 2018, 61, 140-151. [CrossRef]

8. Liang, C.; Fan, L.; Xu, D.; Ding, Y.; Gen, M. Research on coupling scheduling of quay crane dispatch and configuration in the container terminal. Comput. Ind. Eng. 2018, 125, 649-657. [CrossRef] 
9. Msakni, M.K.; Diabat, A.; Rabadi, G.; Al-Salem, M.; Kotachi, M. Exact methods for the quay crane scheduling problem when tasks are modeled at the single container level. Comput. Oper. Res. 2018, 99, 218-233. [CrossRef]

10. Kim, K.H.; Park, Y.M. A crane scheduling method for port container terminals. Eur. J. Oper. Res. 2004, 156, 752-768. [CrossRef]

11. Nguyen, S.; Zhang, M.; Johnston, M.; Tan, K.C. Hybrid evolutionary computation methods for quay crane scheduling problems. Comput. Oper. Res. 2013, 40, 2083-2093. [CrossRef]

12. Zhang, Z.; Liu, M.; Lee, C.Y.; Wang, J. The quay crane scheduling problem with stability constraints. IEEE Trans. Autom. Sci. Eng. 2018, 15, 1399-1412. [CrossRef]

13. Al-Dhaheri, N.; Diabat, A. A Lagrangian relaxation-based heuristic for the multi-ship quay crane scheduling problem with ship stability constraints. Ann. Oper. Res. 2017, 248, 1-24. [CrossRef]

14. Kim, K.H.; Bae, J.W. A look-ahead dispatching method for automated guided vehicles in automated port container terminals. Transp. Sci. 2004, 38, 224-234. [CrossRef]

15. Choe, R.; Kim, J.; Ryu, K.R. Online preference learning for adaptive dispatching of AGVs in an automated container terminal. Appl. Soft Comput. 2016, 38, 647-660. [CrossRef]

16. Kim, J.; Choe, R.; Ryu, K.R. Multi-objective optimization of dispatching strategies for situation-adaptive AGV operation in an automated container terminal. In Proceedings of the 2013 Research in Adaptive and Convergent Systems, Montreal, QC, Canada, 1-4 October 2013.

17. Singgih, I.K.; Hong, S.; Kim, K.H. Flow Path Design for Automated Transport Systems in Container Terminals Considering Traffic Congestion. Ind. Eng. Manag. Syst. 2016, 15, 19-31. [CrossRef]

18. Legato, P.; Mazza, R.M.; Trunfio, R. Simulation-based optimization for discharging/loading operations at a maritime container terminal. OR Spectrum 2010, 32, 543-567. [CrossRef]

19. Xin, J.; Negenborn, R.R.; Lodewijks, G. Energy-aware control for automated container terminals using integrated flow shop scheduling and optimal control. Transp. Res. Part C Emerg. Technol. 2014, 44, 214-230. [CrossRef]

20. Peng, Y.; Wang, W.; Liu, K.; Li, X.; Tian, Q. The Impact of the allocation of facilities on reducing carbon emissions from a green container terminal perspective. Sustainability 2018, 10, 1813. [CrossRef]

21. Yang, Y.; Zhu, X.; Haghani, A. multiple equipment integrated scheduling and storage space allocation in rail-Water intermodal container terminals considering energy efficiency. Transp. Res. Rec. 2019, 2673, 199-209. [CrossRef]

22. Dkhil, H.; Yassine, A.; Chabchoub, H. Optimization of container handling systems in automated maritime terminal. In Advanced Methods for Computational Collective Intelligence; Springer: Berlin/Heidelberg, Germany, 2013; pp. 301-312.

23. Yang, Y.; Zhong, M.; Dessouky, Y.; Postolache, O. An integrated scheduling method for AGV routing in automated container terminals. Comput. Ind. Eng. 2018, 126, 482-493. [CrossRef]

24. Xin, J.; Negenborn, R.R.; Corman, F.; Lodewijks, G. Control of interacting machines in automated container terminals using a sequential planning approach for collision avoidance. Transp. Res. Part C Emerg. Technol. 2015, 60, 377-396. [CrossRef]

25. Lee, D.H.; Wang, H.Q.; Miao, L. Quay crane scheduling with non-interference constraints in port container terminals. Transp. Res. Part E Logist. Transp. Rev. 2008, 44, 124-135. [CrossRef]

26. Baldi, M.M.; Perboli, G.; Tadei, R. The three-dimensional knapsack problem with balancing constraints. Appl. Math. Comput. 2012, 218, 9802-9818. [CrossRef]

27. Luo, X. Comparison of horizon transportation system of full automatic container terminal. Port Waterw. Eng. 2016, 9, 76-82.

28. Chen, C.; Zhang, Z.; Zeng, Q.C. Integrated scheduling model of mixed cross-operation for container terminal. J. Traffic Transp. Eng. 2012, 3, 92-100.

29. Han, X.; Fan, J. Analysis of AGV dispatching and configuration simulation of automated container terminals. J. Chongqing Jiaotong Univ. 2016, 35, 151-154, 164.

(C) 2019 by the authors. Licensee MDPI, Basel, Switzerland. This article is an open access article distributed under the terms and conditions of the Creative Commons Attribution (CC BY) license (http://creativecommons.org/licenses/by/4.0/). 